\title{
White matter degeneration in remote brain areas of stroke patients with motor impairment due to basal ganglia lesions
}

\section{Xuejin Cao}

Southeast University https://orcid.org/0000-0002-3684-8092

\section{Zan Wang}

Southeast University, Zhongda hosiptial

\section{Xiaohui Chen}

Southeast University Zhongda Hospital

Yanli Liu

Southeast University Zhongda Hospital

Wei Wang

Southeast University Zhongda Hospital

Idriss Ali Abdoulaye

Southeast University

Xi Yang

Southeast University Zhongda Hospital

Shiyao Zhang

Southeast University

\section{Xiaoying Guo}

Southeast University

\section{Shanshan Wu}

Southeast University

Yijing Guo ( $\nabla$ guoyijingseu@126.com )

Southeast University https://orcid.org/0000-0002-6638-8963

\section{Research Article}

Keywords: Ischemic stroke, Motor impairment, Magnetic resonance imaging, Diffusion tensor imaging, White matter structural network.

Posted Date: July 16th, 2021

DOl: https://doi.org/10.21203/rs.3.rs-36124/v4 
License: (c) (i) This work is licensed under a Creative Commons Attribution 4.0 International License. Read Full License

Version of Record: A version of this preprint was published at Human Brain Mapping on July 7th, 2021. See the published version at https://doi.org/10.1002/hbm.25583. 


\section{Abstract}

Diffusion tensor imaging (DTI) studies have revealed distinct white matter characteristics of the brain following diseases. Beyond the lesion-symptom maps, stroke is characterized by extensive structural and functional alterations of brain areas remote to local lesions. Here, we further investigated the structural changes over a global level by using DTI data of ten ischemic stroke patients showing motor impairment due to basal ganglia lesions and 11 healthy controls. DTI data were processed to obtain fractional anisotropy (FA) maps, and multivariate pattern analysis (MVPA) was used to explore brain regions that play an important role in classification based on FA maps. The white matter (WM) structural network was constructed by the deterministic fiber-tracking approach. In comparison with the controls, the stroke patients showed FA reductions in the perilesional basal ganglia, brainstem, and bilateral frontal lobes. Using network-based statistics (NBS), we found a significant reduction in the WM subnetwork in stroke patients. We identified the patterns of WM degeneration affecting brain areas remote to the lesions, revealing the abnormal organization of the structural network in stroke patients, which may be helpful in understanding of the neural mechanisms underlying hemiplegia.

\section{Background}

Focal brain lesions can affect the overall performance of brain networks. Over the past decades, numerous neuroimaging studies using magnetic resonance imaging (MRI) have investigated the structural and functional reorganizations after stroke (Lim \& Kang, 2015). Diffusion tensor imaging (DTI) is commonly used to evaluate the structural integrity of the white matter (WM). Previous DTI studies in stroke patients have calculated several diffusion tensor (DT) indicators (e.g., fractional anisotropy [FA], mean diffusivity [MD], radial diffusivity [RD]) in various regions along the corticospinal tract (CST) of the lesioned and contralateral hemispheres (Cunningham et al., 2015; Visser et al., 2018), or computed the CST integrity (Byblow, Stinear, Barber, Petoe, \& Ackerley, 2015; Feng et al., 2015). These imaging indicators were subsequently correlated with functional outcomes, further establishing their value as predictive markers. Certain correlations have been identified between changes in specific fiber bundles and functional outcomes, such as aphasia (Meier, Johnson, Pan, \& Kiran, 2019), neglect (Umarova et al., 2017), and paralysis (Jang et al., 2014). One study measured FA and axial diffusivity (AD) across 48 different WM tract regions in the brains of five hemiparetic patients. They found that patients with lesions involving the corona radiata and middle cerebral artery showed widespread reductions in perilesional FA and described longitudinal changes in the perilesional and remote FA and $A D$ in relation to kinematic parameters of elbow flexion in the subacute post-stroke period (Oey et al., 2019). This globally reduced FA after stroke may reflect extensive Wallerian degeneration (WD) of the descending pathways. Another study investigated global alterations of the lesion-spared network architecture in the acute and chronic stroke phases in patients showing spatial neglect. In addition to anterograde and retrograde axonal degeneration, structural network alterations can represent remote remodeling of fibers not directly connected to the lesion, i.e., transneuronal degeneration. Moreover, the results showed that longitudinal 
white matter changes, also transneuronal changes, may follow the persisting deficit (Umarova et al., 2017).

Unlike the CST command of motor functions, the involvement of other fibers and brain areas in movement control is not well understood. Voluntary movements are mostly controlled by the CST. The primary motor cortex is the source of most corticospinal axons, but its activity is strongly influenced by the pallidum, striatum, cerebellum, and many other cortical regions, including the somatosensory area of the cortex. Different cortical regions are involved in specific motor functions, such as motor learning, motor planning, motor preparation, and coordination. A complete action includes motor initiation and termination, feed-forward and feed-back control loops, and feedback processing. Central processing systems also regulate movement through integration of sensory information with the motor plan. When the CST is damaged by large forebrain lesions in humans, the loss of fine movement is accompanied by hypertonia and hyperreflexia. These deficits do not appear in monkeys with lesions restricted to the CST, so they are probably attributable to damage to adjacent forebrain structures, such as the striatum, in humans (Watson et al., 2010; Desrochers et al., 2019). Therefore, neural degeneration in some regions may cause specific functional manifestations. We performed a pattern-recognition classification of FA maps of the brain in an attempt to explore regions that are closely related to motor function.

Multivariate pattern analysis (MVPA) is an imaging data analysis method based on machine learning and pattern recognition. It involves the use of pattern-classification algorithms to extract spatial patterns from neuroimaging data for the analysis of individual characteristics (Lao et al., 2004). MVPA offers the advantage of considering interregional correlations and searching for abnormalities throughout the entire brain because it adopts an unbiased and whole-brain method without artificially setting the region of interest (ROI) (Pereira, Mitchell, \& Botvinick, 2009). This method has been widely used in psychiatryrelated studies to analyze mild changes observable in subclinical populations (Li et al., 2014; Janssen, Mourão-Miranda, \& Schnack, 2018).

White matter structural connectivity can be modeled as a network. A network-based statistics (NBS) method can control the family-wise error rate during mass univariate testing of every connection of the network. NBS can be also used to investigate the interregional correlations of the brain on a global level (Fortanier et al., 2019; Zalesky, Fornito, \& Bullmore, 2010). We utilized these methods to explore subtle relevant changes and abnormalities of structural networks of the brain for a deeper understanding of the intrinsic brain structural basis of residual motor dysfunctions in ischemic stroke patients.

\section{Methods}

\section{Participants}

Ten right-handed stroke patients (mean age, $56.7 \pm 10.5$ years) from the Southeast University-affiliated Zhongda Hospital were recruited for this study from March 2019 to December 2019. The inclusion criteria for patients were as follows:(1) age $\geq 20$ and $\leq 80$ years; (2) first onset of ischemic stroke with the involvement of the basal ganglia; (3) pure motor deficits; and (4) stable condition after treatment of acute 
stroke without recurrence. The exclusion factors were as follows: (1) a history of neurological or psychiatric disorders prior or subsequent to symptomatic stroke; (2) brain abnormalities unrelated to the infarct lesions; and (3) MRI contraindications. All of the affected extremities were evaluated for motor function. The motor outcome of the affected limbs was evaluated by the Fugl-Meyer assessment (FMA), including the upper and lower extremities (Feng et al., 2015). The Brunnstrom stage (BRS) was also recorded. Recovery of the affected extremities was scored on a 6-point scale ( $1=$ severe; $6=$ normal $)$ (Naghdi, Ansari, Mansouri, \& Hasson, 2010). The clinical characteristics of the stroke patients are summarized in Table 2. Eleven demographically matched healthy control participants (mean age, $61.5 \pm$ 7.8 years) were also recruited.

The study was approved by the local Ethics Committee of the Southeast University-affiliated Zhongda Hospital. All participants provided written informed consent to participate in accordance with the Declaration of Helsinki.

\section{Image acquisition}

Diffusion-tensor images were acquired using a 3.0-Tesla Philips (Ingenia) Medical System equipped with a Synergy-L Sensitivity Encoding (SENSE) head coil and a single echo planar imaging (EPI) sequence, and 33 diffusion-weighted images $\left(b=1000 \mathrm{~s} / \mathrm{mm}^{2}\right)$ and a reference T2-weighted image with no diffusion weighting $\left(b=0 \mathrm{~s} / \mathrm{mm}^{2}\right)$ were obtained with the following acquisition parameters: voxel size = 2 $\times 2 \times 2 \mathrm{~mm}^{3}$, gap = $0 \mathrm{~mm}$; echo time $(\mathrm{TE})=107 \mathrm{~ms}$; repetition time $(\mathrm{TR})=5835 \mathrm{~ms}$; field of view $(\mathrm{FOV})=$ $256 \times 256 \mathrm{~mm}^{2} ;$ flip angle $(\mathrm{FA})=90^{\circ} ;$ matrix $=128 \times 128 ;$ and slices $=75$.

High-resolution T1-weighted axial images covering the whole brain were obtained by a 3D-magnetization prepared rapid gradient-echo (MP-RAGE) sequence with the following parameters: TR $=9.6 \mathrm{~ms} ; \mathrm{TE}=3.7$ $\mathrm{ms} ; \mathrm{FA}=9^{\circ} ;$ matrix $=256 \times 256 ; \mathrm{FOV}=256 \times 256 \mathrm{~mm}^{2} ;$ voxel size $=1 \times 1 \times 1 \mathrm{~mm}^{3} ;$ gap $=0 \mathrm{~mm}$; and number of slices $=140$. Additionally, sagittal fluid attenuated inversion recovery (FLAIR) images were obtained with the following parameters: $\mathrm{TE}=110 \mathrm{~ms}$; $\mathrm{TR}=7000 \mathrm{~ms}$; inversion time $(\mathrm{TI})=2200 \mathrm{~ms}$; $\mathrm{FA}=$ $90^{\circ}$; matrix size $=480 \times 480 ; \mathrm{FOV}=250 \times 250 \mathrm{~mm}^{2}$; slice thickness $=5 \mathrm{~mm}$; number of slices $=20$.

\section{Lesion mapping}

Lesion-side normalization was performed to place lesions on the left side of the brain. For patients with lesions in the right hemisphere, the images were flipped from the right to the left along the midsagittal line to simplify the comparison with other patients with stroke. In accordance with previous investigations, lesion masks of each patient were manually segmented on individual structural MRI images (T1-weighted MP-RAGE and FLAIR images) using MRIcron software (http://www.mricro.com). After spatial normalization of all individual lesion masks, a lesion overlap image for all patients was constructed (Fig. 1) (Grefkes et al., 2008; Chen \& Schlaug, 2013; Zhang et al., 2016).

\section{DTI data pre-processing and network definition}


DTI data analysis was performed by a pipeline toolbox for analyzing brain diffusion images (PANDA, http://www.nitrc.org/projects/panda) (Cui, Zhong, Xu, He, \& Gong, 2013). The main procedure includes the following steps: (1) correction for head motion and eddy current effects using FMRIB's Diffusion Toolbox (FDT); (2) calculation of the DT metric, i.e., FA, for each voxel by using the DTIFIT tool; and (3) normalization by registration of all the individual FA images to the FMRIB58_FA template by calling the FNIRT tool (Guo et al., 2019). The FA maps were obtained, and the FA values of 50 WM labels were computed according to the WM atlas "rICBM_DTI_81_WMPM_FMRIB58.nii.gz." This atlas is created by hand-segmenting a standard-space average of diffusion MRI tensor maps from 81 normal participants according to histology criteria and consists of 50 core regions (Mori et al., 2008).

Whole-brain tractography for each participant was performed in the native diffusion space based on fiber assignment by the continuous tracking (FACT) algorithm (Mori et al., 1999). All voxels with fractional anisotropy ( $F A$ ) values $\geq 0.2$ were used as seed points; the FA and curvature thresholds of path tracing were set to $0.2^{\circ}$ and $45^{\circ}$, respectively.

FA-weighted networks were constructed using 116 nodes defined according to the Automated Anatomical Labeling (AAL) atlas (Tzourio et al., 2002). The entire cerebral cortex was automatically partitioned into 116 anatomical regions of interest (ROIs) (45 ROls for each cerebral hemisphere, $26 \mathrm{ROIs}$ for the cerebellum) using the AAL algorithm. The weight of the edges in the network was defined as the mean FA value of the connected fibers between each pair of nodes. In order to reduce false-positive connections, two nodes were considered structurally connected only when at least 3 fibers were reconstructed between them (Shu et al., 2011).

\section{Multivariate pattern analysis}

Pattern classification analysis could be used to examine the differences in the FA values between groups. A specific MVPA approach known as support vector machine (SVM) was implemented using the Pattern Recognition for Neuroimaging toolbox (PRoNTo) software (http://www.mlnl.cs. ucl.ac.uk/pronto/) (Schrouff et al., 2013). Individual FA maps were treated as points located in a high dimensional space. A linear decision boundary in this high dimensional space was defined by a hyperplane that separated the individual brain images according to a class label (i.e., patients vs. controls) (Fig. 2A). A more detailed description of the SVM can be found in previous reports (Li et al., 2014). The receiver operating characteristic curve (ROC), sensitivity, and specificity of the FA classifications and the weight of each brain region in the classification analysis were obtained. The brain regions with voxels showing values $\geq$ $30 \%$ of the maximum weight vector value of the discrimination map were considered to be the key areas.

\section{Statistical analysis}

Two-sample $t$ tests were employed to test the group differences in demographic data and imaging measures, while the differences in FA values of 50 WM labels were compared between the two groups by using the $t$ test. Correlations between FA values in the core regions and clinical scores were computed using Pearson's correlation test. These statistical tests were performed using the Statistical Package for 
Social Science (SPSS) 22.0 software. The two-sample $t$ test was adopted for the comparisons of internodal connections, followed by NBS using GRETNA (v2.0.0) (Wang et al., 2015) to analyze the FA networks between groups. In addition, the backbone extraction for the structural connectivity matrix (the subnetwork) of each group was calculated to show the group probability matrix. Visualization of the results was performed by the BrainNet Viewer (Xia, Wang, \& He, 2013). The comparisons of the two sets of FA values in the key areas derived from MVPA were performed in SPM12 with false discovery rate $($ FDR $)$ correction $\left(P_{F D R}<0.05\right.$, cluster size $\left.>5\right)$ (Liang et al., 2020).

\section{Results}

\section{Demographic data and patient characteristics}

The demographic data of the patients and the controls are presented in Table_1_Supplnfo. No significant differences in age or sex were observed between the two groups. The mean interval from stroke onset to DTI scans was $10.3 \pm 9.0$ weeks (Table 1 ). Stroke lesions were projected to the left hemisphere for each patient and overlaid onto a T1 template in MNI standard space (Fig. 1).

WM labels with significantly different FA values between control and stroke groups are shown in Table 3 . The WM labels that correlated with movement scores were evaluated with $r$ values. Except for the FA of the fornix, which increased, all other FA values reduced in the stroke group. The most significant WM labels that showed correlation with FMA $(p<0.001)$ were the left posterior limb of internal capsule (PLIC, $r=0.795)$, superior corona radiata (SCR, $r=0.720$ ), and superior fronto-occipital fasciculus (SFOF, $r=$ $0.744)$.

\section{Overall classifier performance}

Figure $2 \mathrm{~A}$ shows the results of the MVPA classification between the 10 stroke patients and the 11 controls based on FA values. The overall accuracy was $100 \%$, and was significant at $P<0.005(P=$ 0.001). Both the sensitivity and the specificity were $100 \%$ (with ROC shown in Fig. 2B). The overall classification accuracy of the algorithm measures its ability to correctly sort the two groups.

\section{Discrimination map}

In the whole-brain voxel weight maps, the weight vector value indicates the relative importance of the voxel in the decision function, i.e., the discrimination between patients and controls (Fig. 3). Note that all voxels in the white matter mask contribute to the decision function since the analysis is multivariate. The spatial distribution of the weight vector provided information about the contribution of different areas to classification.

The brain regions that contributed the most to the discrimination between stroke patients and controls were identified by setting the threshold to $\geq 30 \%$ of the maximum weight vector scores, consistent with previous studies using MVPA for disease classification (Ecker et al., 2010; Li et al., 2014). The most 
frequently identified classifying features of the FA maps included the perilesional basal ganglia and brainstem, with a few features appearing in the bilateral frontal lobes (Fig. 3 and 4 A), which were considered as the key areas.

By comparing the FA values of the two groups in the discrimination map by SPM12 ( $P_{\mathrm{FDR}}<0.05$, cluster size $>5$ ), we found that the stroke group showed reduced FA in the key areas, as shown in Figure $4 \mathrm{~A}$ and reported in Table 2. According to the white matter atlas, these clusters were mainly located in the CST pathway. Moreover, the FA values of these areas showed a positive correlation with that of the ipsilesional CST ( $r=0.888, p<0.001)$ (Fig. 4 B).

\section{Decreased connection of the component network in stroke patients}

FA-weighted networks were constructed from the nodes (brain areas) defined according to the AAL atlas. The weight of the edges in the network was defined as the mean FA value of the connected fibers between each pair of nodes. The NBS approach was used on the structural networks constructed by deterministic tractography. We identified several significantly decreased connections of a component network (subnetwork) in stroke patients $(P<0.001, P=0.00099)$ (Fig. $5 \mathrm{~A}$ and Table_2_Supplnfo).

Figure $5 \mathrm{~A}$ presents the subnetwork that showed deterioration in the brain structure of the stroke patients. Figures $5 \mathrm{~B}$ and $\mathrm{C}$ demonstrate the group probability matrix for the subnetwork of each group. The table in supplementary information reported the regions as nodes in the subnetwork. The subnetwork contained parts of the cerebral cortical regions (the frontal, parietal, and occipital lobes), the subcortical areas (the basal ganglion), and the cerebellum. Together with the results of MVPA, these findings showed obvious changes involving the frontal parietal lobe and the basal ganglia, and those in the brainstem region may overlap with some of the fibers connected to the cerebellum.

nt characteristics

The demographic data of the patients and the controls are presented in Table 1. No significant differences in age or gender were observed between the two groups. The mean interval from stroke onset to DTI scans was $10.3 \pm 9.0$ weeks (Table 2 ). The lesions were mainly located in the basal ganglia region. For the 3 patients with lesions in the right hemisphere, the imaging data were laterally flipped. Stroke lesions for each patient were overlaid onto a T1 template in MNI standard space (Fig. 1).

The FA values of the ipsilesional CST in the patients were statistically significant reduced than that of the contralateral side $(t=5.257, P=0.001)$. Compared with the controls, the FA values of the left (injured side) CST in the stroke group were significantly decreased $(t=6.511, P<0.001)$. And no significant differences in the FA values of the right CST $(t=0.153, P=0.88)$ were observed between the two groups.

\section{Overall Classifier Performance}

Figure $2 \mathrm{~A}$ shows the result of the MVPA classification between the 10 stroke patients and the 11 controls based on FA values. The overall accuracy was $100 \%$, and was significant at $P \leq 0.001(P=0.001)$. Both 
the sensitivity and the specificity were $100 \%$ (with ROC shown in Fig. 2B). This overall classification accuracy of the algorithm measures its ability to correctly sort the two groups.

\section{Discrimination Map}

In the whole-brain voxel weight maps, the weight vector value indicates the relative importance of the voxel in the decision function -- discrimination between patients and controls (Fig. 3). Note that all voxels in the white matter mask contribute to the decision function since the analysis is multivariate. The spatial distribution of the weight vector provided information about the contribution of different areas to classification.

The brain regions that contributed the most to the discrimination between stroke patients and controls were identified by setting the threshold to $\geq 30 \%$ of the maximum weight vector scores, consistent with previous studies using MVPA for disease classification [11,29]. The most identified classified features of the FA maps included the perilesional basal ganglia and brainstem, with a few in the bilateral frontal lobes (Fig. 3 and 4 A), which were taken as the key areas.

By comparing the FA values of the two groups in the discrimination map by SPM12 $\left(P_{\mathrm{FDR}}<0.05\right.$, cluster size $>5$ ), we found that the stroke group showed reduced FA in the key areas, shown in Figure 4A and reported in Table 3. According to the white matter atlas, the clusters were mainly located in the CST pathway. Moreover, the FA values of these areas have a positive correlation with that of the ipsilesional CST ( $r=0.888, p<0.001)$ (Fig. 4 B).

\section{Decreased Connection of the Component Network in stroke patients}

FA weighted networks were constructed from the nodes (brain areas) defined according to the AAL atlas. The weight of the edges in the network was defined as the mean FA value of the connected fibers between each pair nodes. The NBS approach was used on the structural networks constructed by deterministic tractography. We identified several significantly decreased connections of a component network (subnetwork) in stroke patients $(P<0.01, P=0.008)$ including 26 regions and 32 connections (Table 4 and Fig. 5 A).

Figure 5 showed the subnetwork that reveals deterioration in the brain structure of the stroke patients. Table 3 reported the regions as nodes in the subnetwork. The backbone matrix of the group structural matrixes for each group of the subnetwork was calculated, including the 26 regions in Table 4 (Fig. 5 B and C). The subnetwork was composed of the cerebral cortical regions (the frontal lobe, the parietal lobe and the occipital lobe), the subcortical areas (the basal ganglion), and parts of the cerebellum. Compared with the results of MVPA, there were changes involving the frontal parietal lobe and the basal ganglia, and those in the brainstem region may overlap with some of the fibers connected to the cerebellum.

\section{Discussion}


Beyond the well-known concept of lesion-symptom mapping, some lesions in a single location in the brain could disrupt brain functions routed to widespread neural networks (Burke et al., 2015; Lim, et al., 2014). Our analysis confirmed that local destruction of basal ganglia could affect remote areas in the brain.

\section{White matter degeneration in the CST pathway of stroke patients}

The degree of anisotropy depends on the level of organization, the integrity of the white matter tract, and the degree of freedom for water diffusion caused by the oriented axonal membranes and myelin sheaths (Virta, Barnett, \& Pierpaoli, 1999). Reduced anisotropy along the CST far from the original lesions has been interpreted as WD (Thomalla et al., 2004). DTI can quantify the FA values to evaluate pathology changes in the white matter, such as WD. Using MVPA, we reported that apart from the basal ganglia region where the infarcts are localized, brain areas with significantly decreased FA values were also located in the brainstem of the lesioned hemisphere, and a few areas were present in the bilateral frontal lobes, which might be indicative of the degenerative lesions caused by WD.

The acute and chronic phases of stroke probably differ in terms of WM changes since neural changes can include anterograde and retrograde degeneration, or refactoring. However, regardless of the type of alteration, it should generate specific structural changes and affect the corresponding functions.

Therefore, we performed pattern-recognition classification using the whole-brain FA map and explored the key brain regions important for distinguishing stroke patients from controls. MVPA analysis explored the key brain regions important for distinguishing stroke patients from controls. Most of the regions were located in the WM based on the atlas, but some voxels were still not in the WM areas. They may have been present at the junction of gray and white matter. Therefore, we can investigate the areas that were embedded in a network by NBS. In comparison with the whole brain network, we referred to the decreased FA-weighted component network as a subnetwork. Its contents are displayed in Figure 5 , and include the frontal lobe, limbic lobe, occipital lobe, parietal lobe, basal ganglia, temporal lobe, and cerebellum. We thought that this subnetwork may be specific to basal ganglia stroke and could be generalizable for patients with hemiplegia.

Our study verified the degenerative changes in the WM of stroke patients. The infarcted lesions were mainly located in the basal ganglia region, but the FA reduction in some remote areas had reached the point where they could be differentiated from the controls. Thus, the damaged structural anatomy of the subcortical areas may induce deterioration of key WM areas in the brain.

\section{Decreased WM connections were widely distributed across the brain regions of stroke patients}

Many patients showed motor dysfunctions after the occurrence of cerebral infarction. These dysfunctions were usually related to injury of the CST. The CST originates from multiple motor and somatosensory cortices, including the premotor cortex, supplementary motor cortex (SMA), primary motor cortex, as well as primary and secondary somatosensory cortices. The CST is crucial for proper execution of a volitional movement (Lemon, 2008; Chenot et al., 2019). Apart from the CST and the motor areas of 
the cortex, the proper execution of movements involving balance and coordination also requires the extrapyramidal tract and other brain regions such as basal ganglia and the cerebellum (Moreno-López et al., 2016). The NBS analysis showed that the structural subnetwork connection of the stroke group was weaker than that of the control group, indicating that the nerve fibers involved in the subnetwork were affected. Destruction of the integrity and order of the brain structure may be reflected in the WM. Not only was the motor cortex directly related to motor commands, but other regions that regulated movement were also involved in the subnetwork.

In addition, since the MVPA analysis is multivariate, all voxels in the WM mask contribute to the decision function in the processing stage. The CST passes through the frontal lobe and midbrain, and MVPA verified its pathway degeneration in stroke patients with hemiplegia. Other brain areas influenced were explored by NBS (e.g., the occipital lobe, parietal lobe, and cerebellum). The results of MVPA more obviously showed the damage to the pathway from the basal ganglia to the brain stem (Fig. 4). The results of NBS showed that the affected brain tissues were distributed across a wide range of brain regions, and the damage to the pathway from the basal ganglia to the cortex was more obvious (Fig. 5). Both methods showed reduced FA in the frontal lobe and basal ganglia. The connections between the frontal lobe and the cerebellum pass through the cerebral peduncle in the brainstem. Therefore, the weakening of these connections in subnetworks derived from NBS was partially consistent with the decreased FA in the brainstem based on MVPA. The affected contralesional areas were verified with the difference of the corpus callosum and fornix between groups. In short, the decreased FA appears to spread out from the original infarct area as time elapses after the acute stage of the stroke onset. The subnetwork specific to basal ganglia stroke implicated the involvement of an internal model in patients with hemiplegia. Next, we briefly discuss the brain regions involved in the subnetwork.

\section{The bilateral frontal lobe}

Similar to the results of previous studies showing infarct-related focal thinning of the motor area in the remote cortex via degeneration of inter-hemispheric connection fibers of the corpus callosum (Duering et al., 2015; Hayward et al., 2017), we found changes in the connection between the frontal hemispheres, as well as reduced FA values in a small area located in the contralesional frontal cortex. In studies calculating the mean kurtosis (MK) values of manually drawn ROIs from diffusion kurtosis imaging (DKI), secondary degeneration has been reported to occur in the ipsilesional precentral gyrus at the 6-month follow-up after subcortical stroke involving the CST (Wei, Shang, Zhou, Zhou, \& Li, 2019).

In Figure 5, the connection between the left precentral gyrus (PreCG) and middle frontal gyrus (MFG) in the subnetwork derived from NBS was reduced in the stroke group, which may well be linked to reduced FA in the frontal lobe in Figure 4. This cluster was not present in the white matter atlas, but was present in the MFG (Table 2). The CST originates from motor cortices, including the PreCG, premotor cortex, and SMA in MFG. In Table 3, FA values of the superior corona radiata (CR), anterior CR, superior longitudinal fasciculus (SLF), and parts of the internal capsule were shown to be decreased in the stroke group. We 
speculated that the FA changes in the CST pathway and that these changes may be linked with the related motor supplementary areas in frontal lobes.

\section{The basal ganglia region}

The patients recruited in this study all had infarcts localized to the basal ganglia. The comparison results for the WM regions in Table 3 showed that the PLIC, anterior limb of the internal capsule (ALIC), retrolenticular part of internal capsul (RIC), and external capsule (EC) were affected after stroke. These WM tracts were located between the thalamus and basal ganglia. The advantage of the structural subnetwork derived from NBS was that it showed connections between smaller brain areas, such as the edges between the thalamus, putamen, pallidum, PreCG, SMA, and PoCG (Fig. 5B). The CST section passing through the internal capsule may receive regulatory information from the nucleus of basal ganglia. Due to the cortico-basal ganglia-thalamocortical "motor" loop, any impact on the circuit constituents can lead to a shift in the balance between neural interactions in the direct and indirect pathways and subsequently lead to variations in the brain functions (Alexander, Crutcher, \& DeLong, 1991; Silkis, 2001). In the early stage of rehabilitation, stroke patients with hemiplegia often have synergistic movements, which are thought to be related to this loop.

\section{The parietal and occipital lobes}

Fibers connecting the frontal, parietal, and occipital lobes were affected after stroke (Fig. 5), and the SFOF showed obvious degeneration in the stroke group (Table 3). The parietal lobe participates in sensory and motor integration, while the occipital lobe is related to visual effects. Stroke patients with dysfunction of normal voluntary movements may develop corresponding abnormal sensory modulations that gradually affect brain structure (Buaron, Reznik, Gilron, \& Mukamel, 2020).

\section{The cerebellum}

The cerebellum is often related to balance adjustment, and patients with hemiplegia usually experience problems with stability and coordination after stroke. The AAL atlas did not contain regions in the brainstem. Therefore, connections in the network could not precisely demonstrate fibers to the brainstem, including the red nucleus and substantia nigra. In our study, the cerebral peduncle (CP) was changed after stroke, and the reduced FA of the brainstem was probably related to changes in cortico-pontocerebellar tract (CPCT). Previous DTI studies also showed decreased FA in the midbrain of stroke patients by manually plotting ROls (Wei et al., 2019). A recent study indicated that the cerebellum plays a role in residual motor output by facilitating cortical excitability in chronic stroke (Guder et al., 2020). Taken together, these findings suggest that the inability to perform normal movements might gradually lead to abnormal balance, which is reflected in decreased cortico-cerebellar connectivity in these patients.

\section{Limitations}

This study had multiple limitations. First, the sample size was small. Second, the interval of DTI acquisition from stroke onset ranged from 2 to 24 weeks. Recovery of FA in the penumbra regions occurs 
most rapidly during the first two weeks following stroke, with continued slow increases in FA occurring for many weeks thereafter (Ding et al., 2008; Mandeville, Ayata, Zheng, \& Mandeville, 2016). The phases of rehabilitation may influence white matter organization, but we only discussed stroke patients with motor impairments in a cross-sectional manner. Future studies should aim to further explore these findings by expanding the sample size and dynamically observing changes from the acute to chronic phases. Additionally, emphasis should be placed on the classification and refinement of clinical behaviors of stroke patients, with identification of the specific brain feature changes that correspond to the functional outcomes.

\section{Conclusions}

Our study recruited stroke patients with motor dysfunction and used MVPA and NBS methods based on DTI data to detect reduced FA values and abnormal white matter connections at a global level in the brain of these patients. We found multiple WM structural abnormalities in the affected brain areas of stroke patients showing motor impairment. Our study may provide the basis for further exploration of the neural mechanisms involved in residual motor deficits in stroke patients. In future studies, we will further compare the neural changes in well-recovered patients to provide a basis for the development of adaptive rehabilitation training strategies.

\section{Abbreviations}

DTI: Diffusion tensor imaging; MVPA: multivariate pattern analysis; NBS: network-based statistic; AAL: Automated Anatomical Labeling; FA: fractional anisotropy; MRI: Magnetic Resonance Imaging; SVM: support vector machine; WM: white matter; MD: mean diffusivity; RD: radial diffusivity; CST: corticospinal tract; ROI: region of interest ; MNI: Montreal Neurological Institute; FMA: Fugl-Meyer assessment; BRS: Brunnstrom stage; WD: Wallerian degeneration; DKI: Diffusion kurtosis imaging; RMET: Reading the Mind in the Eyes Test; FC: functional connectivity.

\section{Declarations}

\section{Acknowledgements}

We thank all the patients and volunteers for participating in this study.

\section{Conflict of interest disclosure}

The authors declare that they have no competing interests.

\section{References}

Alexander, G. E., Crutcher, M. D., \& DeLong, M. R. (1991). Chapter 6 Basal ganglia-thalamocortical circuits: Parallel substrates for motor, oculomotor, "prefrontal" and "limbic" functions. In Progress in Brain 
Research (Vol. 85, pp. 119-146). Elsevier. https://doi.org/10.1016/S0079-6123(08)62678-3

Buaron, B., Reznik, D., Gilron, R., \& Mukamel, R. (2020). Voluntary actions modulate perception and neural representation of action-consequences in a hand-dependent manner. Cerebral Cortex (New York, N. Y.: 1991), 30, 6097-6107. https://doi.org/10.1093/cercor/bhaa156

Burke Quinlan, E., Dodakian, L., See, J., McKenzie, A., Le, V., Wojnowicz, M., ... Cramer, S. C. (2015). Neural function, injury, and stroke subtype predict treatment gains after stroke. Annals of Neurology, 77, 132145. https://doi.org/10.1002/ana.24309

Byblow, W. D., Stinear, C. M., Barber, P. A., Petoe, M. A., \& Ackerley, S. J. (2015). Proportional recovery after stroke depends on corticomotor integrity. Annals of Neurology, 78, 848-859.

https://doi.org/10.1002/ana.24472

Chen, J. L., \& Schlaug, G. (2013). Resting State Interhemispheric Motor Connectivity and White Matter Integrity Correlate with Motor Impairment in Chronic Stroke. Frontiers in Neurology, 4, 178. https://doi.org/10.3389/fneur.2013.00178

Chenot, Q., Tzourio-Mazoyer, N., Rheault, F., Descoteaux, M., Crivello, F., Zago, L., ... Petit, L. (2019). A population-based atlas of the human pyramidal tract in 410 healthy participants. Brain Structure \& Function, 224, 599-612. https://doi.org/10.1007/s00429-018-1798-7

Cui, Z., Zhong, S., Xu, P., He, Y., \& Gong, G. (2013). PANDA: A pipeline toolbox for analyzing brain diffusion images. Frontiers in Human Neuroscience, 7. https://doi.org/10.3389/fnhum.2013.00042

Cunningham, D. A., Machado, A., Janini, D., Varnerin, N., Bonnett, C., Yue, G., ... Plow, E. B. (2015). Assessment of inter-hemispheric imbalance using imaging and noninvasive brain stimulation in patients with chronic stroke. Archives of Physical Medicine and Rehabilitation, 96(4 Suppl), S94-103. https://doi.org/10.1016/j.apmr.2014.07.419

Desrochers, P., Brunfeldt, A., Sidiropoulos, C., Kagerer, F.. Sensorimotor Control in Dystonia. Brain Sci. 2019 Apr 11;9(4):79. https://doi.org/10.3390/brainsci9040079.

Ding, G., Jiang, Q., Li, L., Zhang, L., Zhang, Z. G., Ledbetter, K. A., ... Chopp, M. (2008). Magnetic resonance imaging investigation of axonal remodeling and angiogenesis after embolic stroke in sildenafil-treated rats. Journal of Cerebral Blood Flow and Metabolism: Official Journal of the International Society of Cerebral Blood Flow and Metabolism, 28, 1440-1448. https://doi.org/10.1038/jcbfm.2008.33

Duering, M., Righart, R., Wollenweber, F. A., Zietemann, V., Gesierich, B., \& Dichgans, M. (2015). Acute infarcts cause focal thinning in remote cortex via degeneration of connecting fiber tracts. Neurology, 84 , 1685-1692. https://doi.org/10.1212/WNL.0000000000001502

Ecker, C., Rocha-Rego, V., Johnston, P., Mourao-Miranda, J., Marquand, A., Daly, E. M., ... MRC AIMS Consortium. (2010). Investigating the predictive value of whole-brain structural MR scans in autism: A 
pattern classification approach. Neurolmage, 49, 44-56.

https://doi.org/10.1016/j.neuroimage.2009.08.024

Feng, W., Wang, J., Chhatbar, P. Y., Doughty, C., Landsittel, D., Lioutas, V.-A., ... Schlaug, G. (2015). Corticospinal tract lesion load: An imaging biomarker for stroke motor outcomes. Annals of Neurology, 78, 860-870. https://doi.org/10.1002/ana.24510

Fortanier, E., Grapperon, A.-M., Le Troter, A., Verschueren, A., Ridley, B., Guye, M., ... Zaaraoui, W. (2019). Structural Connectivity Alterations in Amyotrophic Lateral Sclerosis: A Graph Theory Based Imaging Study. Frontiers in Neuroscience, 13, 1044. https://doi.org/10.3389/fnins.2019.01044

Grefkes, C., Nowak, D. A., Eickhoff, S. B., Dafotakis, M., Küst, J., Karbe, H., \& Fink, G. R. (2008). Cortical connectivity after subcortical stroke assessed with functional magnetic resonance imaging. Annals of Neurology, 63, 236-246. https://doi.org/10.1002/ana.21228

Guder, S., Frey, B. M., Backhaus, W., Braass, H., Timmermann, J. E., Gerloff, C., \& Schulz, R. (2020). The Influence of Cortico-Cerebellar Structural Connectivity on Cortical Excitability in Chronic Stroke. Cerebral Cortex (New York, N.Y.: 1991), 30, 1330-1344. https://doi.org/10.1093/cercor/bhz169

Guo, X., Liu, R., Lu, J., Wu, C., Lyu, Y., Wang, Z., ... Tong, S. (2019). Alterations in Brain Structural Connectivity After Unilateral Upper-Limb Amputation. IEEE Transactions on Neural Systems and Rehabilitation Engineering: A Publication of the IEEE Engineering in Medicine and Biology Society, 27, 2196-2204. https://doi.org/10.1109/TNSRE.2019.2936615

Hayward, K. S., Neva, J. L., Mang, C. S., Peters, S., Wadden, K. P., Ferris, J. K., \& Boyd, L. A. (2017). Interhemispheric Pathways Are Important for Motor Outcome in Individuals with Chronic and Severe Upper Limb Impairment Post Stroke. Neural Plasticity, 2017, 4281532.

https://doi.org/10.1155/2017/4281532

Jang, S. H., Kim, K., Kim, S. H., Son, S. M., Jang, W. H., \& Kwon, H. G. (2014). The relation between motor function of stroke patients and diffusion tensor imaging findings for the corticospinal tract. Neuroscience Letters, 572, 1-6. https://doi.org/10.1016/j.neulet.2014.04.044

Janssen, R. J., Mourão-Miranda, J., \& Schnack, H. G. (2018). Making Individual Prognoses in Psychiatry Using Neuroimaging and Machine Learning. Biological Psychiatry. Cognitive Neuroscience and Neuroimaging, 3, 798-808. https://doi.org/10.1016/j.bpsc.2018.04.004

Lao, Z., Shen, D., Xue, Z., Karacali, B., Resnick, S. M., \& Davatzikos, C. (2004). Morphological classification of brains via high-dimensional shape transformations and machine learning methods. Neurolmage, 21, 46-57. https://doi.org/10.1016/j.neuroimage.2003.09.027

Lemon, R. N. (2008). Descending pathways in motor control. Annual Review of Neuroscience, 31, 195218. https://doi.org/10.1146/annurev.neuro.31.060407.125547 
Li, F., Huang, X., Tang, W., Yang, Y., Li, B., Kemp, G. J., ... Gong, Q. (2014). Multivariate pattern analysis of DTI reveals differential white matter in individuals with obsessive-compulsive disorder. Human Brain Mapping, 35, 2643-2651. https://doi.org/10.1002/hbm.22357

Liang, Y., Cai, L., Zhou, X., Huang, H., \& Zheng, J. (2020). Voxel-based analysis and multivariate pattern analysis of diffusion tensor imaging study in anti-NMDA receptor encephalitis. Neuroradiology, 62, 231239. https://doi.org/10.1007/s00234-019-02321-x

Lim, J.-S., \& Kang, D.-W. (2015). Stroke Connectome and Its Implications for Cognitive and Behavioral Sequela of Stroke. Journal of Stroke, 17, 256-267. https://doi.org/10.5853/jos.2015.17.3.256

Lim, J.-S., Kim, N., Jang, M. U., Han, M.-K., Kim, S., Baek, M. J., ... Bae, H.-J. (2014). Cortical hubs and subcortical cholinergic pathways as neural substrates of poststroke dementia. Stroke, 45, 1069-1076. https://doi.org/10.1161/STROKEAHA.113.004156

Mandeville, E. T., Ayata, C., Zheng, Y., \& Mandeville, J. B. (2017). Translational MR Neuroimaging of Stroke and Recovery. Translational Stroke Research, 8, 22-32. https://doi.org/10.1007/s12975-016-0497-z

Meier, E. L., Johnson, J. P., Pan, Y., \& Kiran, S. (2019). The utility of lesion classification in predicting language and treatment outcomes in chronic stroke-induced aphasia. Brain Imaging and Behavior, 13, 1510-1525. https://doi.org/10.1007/s11682-019-00118-3

Moreno-López, Y., Olivares-Moreno, R., Cordero-Erausquin, M., \& Rojas-Piloni, G. (2016). Sensorimotor Integration by Corticospinal System. Frontiers in Neuroanatomy, 10, 24.

https://doi.org/10.3389/fnana.2016.00024

Mori, S., Crain, B. J., Chacko, V. P., \& van Zijl, P. C. (1999). Three-dimensional tracking of axonal projections in the brain by magnetic resonance imaging. Annals of Neurology, 45, 265-269.

https://doi.org/10.1002/1531-8249(199902)45:2<265::aid-ana21>3.0.co;2-3

Mori, S., Oishi, K., Jiang, H., Jiang, L., Li, X., Akhter, K., ... Mazziotta, J. (2008). Stereotaxic white matter atlas based on diffusion tensor imaging in an ICBM template. Neuroimage, 40(2), 570582. https://doi.org/10.1016/j.neuroimage.2007.12.035

Naghdi, S., Ansari, N. N., Mansouri, K., \& Hasson, S. (2010). A neurophysiological and clinical study of Brunnstrom recovery stages in the upper limb following stroke. Brain Injury, 24, 1372-1378. https://doi.org/10.3109/02699052.2010.506860

Oey, N. E., Samuel, G. S., Lim, J. K. W., VanDongen, A. M., Ng, Y. S., \& Zhou, J. (2019). Whole Brain White Matter Microstructure and Upper Limb Function: Longitudinal Changes in Fractional Anisotropy and Axial Diffusivity in Post-Stroke Patients. Journal of Central Nervous System Disease, 11, 1179573519863428. https://doi.org/10.1177/1179573519863428 
Pereira, F., Mitchell, T., \& Botvinick, M. (2009). Machine learning classifiers and fMRI: A tutorial overview. Neurolmage, 45(1 Suppl), S199-209. https://doi.org/10.1016/j.neuroimage.2008.11.007

Schrouff, J., Rosa, M. J., Rondina, J. M., Marquand, A. F., Chu, C., Ashburner, J., ... Mourão-Miranda, J. (2013). PRoNTo: Pattern recognition for neuroimaging toolbox. Neuroinformatics, 11, 319-337. https://doi.org/10.1007/s12021-013-9178-1

Shu, N., Liu, Y., Li, K., Duan, Y., Wang, J., Yu, C., ... He, Y. (2011). Diffusion tensor tractography reveals disrupted topological efficiency in white matter structural networks in multiple sclerosis. Cerebral Cortex (New York, N. Y.: 1991), 21, 2565-2577. https://doi.org/10.1093/cercor/bhr039

Silkis, I. (2001). The cortico-basal ganglia-thalamocortical circuit with synaptic plasticity. II. Mechanism of synergistic modulation of thalamic activity via the direct and indirect pathways through the basal ganglia. Biosystems, 59, 7-14. https://doi.org/10.1016/S0303-2647(00)00135-0

Thomalla, G., Glauche, V., Koch, M. A., Beaulieu, C., Weiller, C., \& Röther, J. (2004). Diffusion tensor imaging detects early Wallerian degeneration of the pyramidal tract after ischemic stroke. Neurolmage, 22, 1767-1774. https://doi.org/10.1016/j.neuroimage.2004.03.041

Tzourio-Mazoyer, N., Landeau, B., Papathanassiou, D., Crivello, F., Etard, O., Delcroix, N., ... Joliot, M. (2002). Automated anatomical labeling of activations in SPM using a macroscopic anatomical parcellation of the MNI MRI single-subject brain. Neurolmage, 15, 273-289.

https://doi.org/10.1006/nimg.2001.0978

Umarova, R. M., Beume, L., Reisert, M., Kaller, C. P., Klöppel, S., Mader, I., ... Weiller, C. (2017). Distinct white matter alterations following severe stroke: Longitudinal DTI study in neglect. Neurology, 88, 1546-1555. https://doi.org/10.1212/WNL.0000000000003843

Virta, A., Barnett, A., \& Pierpaoli, C. (1999). Visualizing and characterizing white matter fiber structure and architecture in the human pyramidal tract using diffusion tensor MRI. Magnetic Resonance Imaging, 17, 1121-1133. https://doi.org/10.1016/S0730-725X(99)00048-X

Visser, M. M., Yassi, N., Campbell, B. C. V., Desmond, P. M., Davis, S. M., Spratt, N., ... Bivard, A. (2019). White Matter Degeneration after Ischemic Stroke: A Longitudinal Diffusion Tensor Imaging Study. Journal of Neuroimaging: Official Journal of the American Society of Neuroimaging, 29, 111-118. https://doi.org/10.1111/jon.12556

Watson, C., Kirkcaldie, M., Paxinos, G. (2010). Chapter 5 - Command and control-the motor systems. The Brain. 2010:55-74.

Wang, J., Wang, X., Xia, M., Liao, X., Evans, A., \& He, Y. (2015). GRETNA: A graph theoretical network analysis toolbox for imaging connectomics. Frontiers in Human Neuroscience, 9, 386. https://doi.org/10.3389/fnhum.2015.00386 
Wei, X.-E., Shang, K., Zhou, J., Zhou, Y.-J., \& Li, Y.-H. (2019). Acute Subcortical Infarcts Cause Secondary Degeneration in the Remote Non-involved Cortex and Connecting Fiber Tracts. Frontiers in Neurology, 10, 860. https://doi.org/10.3389/fneur.2019.00860

Xia, M., Wang, J., \& He, Y. (2013). BrainNet Viewer: A network visualization tool for human brain connectomics. PloS One, 8, e68910. https://doi.org/10.1371/journal.pone.0068910

Zalesky, A., Fornito, A., \& Bullmore, E. T. (2010). Network-based statistic: Identifying differences in brain networks. Neurolmage, 53, 1197-1207. https://doi.org/10.1016/j.neuroimage.2010.06.041

Zhang, Y., Liu, H., Wang, L., Yang, J., Yan, R., Zhang, J., ... Qiu, M. (2016). Relationship between functional connectivity and motor function assessment in stroke patients with hemiplegia: A resting-state functional MRI study. Neuroradiology, 58, 503-511. https://doi.org/10.1007/s00234-016-1646-5

\section{Tables}

Table 1. Patient characteristics

\begin{tabular}{lllllll}
\hline ID & Age (years) & Side & Localization of infarct & BRS & FMA & $\begin{array}{c}\text { Scan time } \\
\text { (week) }\end{array}$ \\
\hline 1 & 49 & R & BG & $3,3,5$ & 58 & 22 \\
2 & 67 & R & BG & $2,1,4$ & 37 & 2 \\
3 & 57 & L & BG, PV & $2,1,4$ & 35 & 4 \\
4 & 51 & L & BG, PV & $3,2,4$ & 41 & 10 \\
5 & 60 & R & BG, CR & $4,4,5$ & 86 & 20 \\
6 & 57 & L & BG, CR & $2,1,5$ & 38 & 3 \\
7 & 74 & L & BG, CR & $5,4,5$ & 82 & 14 \\
8 & 60 & L & BG & $2,1,3$ & 19 & 2 \\
9 & 35 & L & BG & $2,1,3$ & 25 & 3 \\
10 & 57 & L & BG & $5,5,5$ & 89 & 24 \\
\hline
\end{tabular}

Side: the hemisphere of lesions on brain; $\mathrm{IC}=$ internal capsule; $\mathrm{CR}=$ corona radiata; $\mathrm{BG}=$ basal ganglia; PV= periventricular; BRS (Brunnstrom stage): separate functional evaluation of proximal and distal portions of the upper and entire lower extremities; FMA = Fugl-Meyer assessment (full score $=100$ ); scan time: interval of DTI acquisition from stroke onset.

Table 2. The brain areas showing decreased FA in patients in comparison with controls 


\begin{tabular}{|c|c|c|c|c|c|c|}
\hline ID & $\begin{array}{l}\text { Voxel } \\
\text { size }\end{array}$ & $\begin{array}{l}\text { Peak MNI } \\
\text { coordinate }(x, y, z)\end{array}$ & $\begin{array}{l}\text { Peak } \\
\text { intensity }\end{array}$ & Brain regions & $\begin{array}{l}\text { White } \\
\text { regions } \\
\text { (voxel size) }\end{array}$ & matter \\
\hline 1 & 36 & $-4,-36,-46$ & 0.028 & Pons; Medulla.L & CST.L & 20 \\
\hline \multirow[t]{2}{*}{2} & 114 & $-10,-20,-22$ & 0.032 & Midbrain; Pons.L & CP.L & 57 \\
\hline & & & & & CST.L & 53 \\
\hline \multirow[t]{4}{*}{3} & 112 & $-22,-6,16$ & 0.027 & Extra-nuclear; & PLIC.L & 43 \\
\hline & & & & Lentiform & SCR.L & 30 \\
\hline & & & & Nucleus.L & SFOF.L & 15 \\
\hline & & & & & ALIC.L & 12 \\
\hline 4 & 9 & $-14,-20,60$ & 0.019 & MFG.L & $\begin{array}{l}\text { Not in } \\
\text { atlas }\end{array}$ & the \\
\hline 5 & 7 & $24,-18,66$ & 0.023 & MFG.R & $\begin{array}{l}\text { Not in the } \\
\text { atlas }\end{array}$ & \\
\hline
\end{tabular}

ID: the index of the cluster; voxel size: number of voxels in the cluster; Peak MNI coordinate: the location of the voxel with the maximum weight vector scores (also peak intensity) in each cluster. Cluster Locater in PANDA software was used to locate the cluster image according to JHU ICBM-DTI-81 White-Matter Labels. White matter atlas (voxel size): the atlas regions this cluster involves and the quantity of voxels in this cluster overlapped with each atlas region. MFG: medial frontal gyrus; CP: cerebral peduncle; PLIC: posterior limb of the internal capsule; SCR: superior corona radiata; SFOF: superior fronto-occipital fasciculus (could be a part of the anterior internal capsule); ALIC: anterior limb of the internal capsule; L: Left; R: Right.

Table 3. White matter (WM) labels with different FA values between groups 


\begin{tabular}{|c|c|c|c|c|}
\hline \multirow[t]{2}{*}{ ID } & \multirow[t]{2}{*}{ WM label } & \multirow{2}{*}{$\begin{array}{l}t \text {-test } \\
p\end{array}$} & \multicolumn{2}{|c|}{ Correlation test } \\
\hline & & & $p$ & $r$ \\
\hline 1 & Fornix & 0.010 & 0.024 & -0.490 \\
\hline & Decreased FA in the stroke group & & & \\
\hline 2 & Splenium of the corpus callosum & 0.035 & 0.088 & \\
\hline & CST.L & 0.000 & 0.005 & 0.588 \\
\hline 4 & Superior CP.R & 0.001 & 0.032 & 0.468 \\
\hline 5 & Superior CP.L & 0.026 & 0.214 & \\
\hline 6 & CP.L & 0.000 & 0.006 & 0.579 \\
\hline 7 & ALIC.R & 0.014 & 0.048 & 0.436 \\
\hline 8 & ALIC.L & 0.003 & 0.118 & \\
\hline 9 & PLIC.R & 0.040 & 0.065 & \\
\hline 10 & PLIC.L & 0.000 & 0.000 & 0.795 \\
\hline 11 & RIC.R & 0.018 & 0.086 & \\
\hline 12 & RIC.L & 0.001 & 0.003 & 0.610 \\
\hline 13 & Anterior CR.L & 0.039 & 0.309 & \\
\hline 14 & Superior CR.R & 0.007 & 0.064 & \\
\hline 15 & Superior CR.L & 0.000 & 0.000 & 0.720 \\
\hline 16 & Posterior CR.R & 0.005 & 0.062 & \\
\hline 17 & Posterior CR.L & 0.003 & 0.038 & 0.456 \\
\hline 18 & Sagittal stratum.R & 0.001 & 0.006 & 0.582 \\
\hline 19 & Sagittal stratum.L & 0.001 & 0.033 & 0.466 \\
\hline 20 & External capsule.L & 0.001 & 0.037 & 0.457 \\
\hline 21 & SLF.R & 0.004 & 0.111 & \\
\hline 22 & SLF.L & 0.007 & 0.110 & \\
\hline 23 & SFOF.L & 0.000 & 0.000 & 0.744 \\
\hline 24 & Uncinate fasciculus.R & 0.008 & 0.179 & \\
\hline
\end{tabular}

WM labels with Significantly different FA values between the control and stroke groups using the $t$-test $(p<0.05)$. The correlation between FA values and FMA scores was determined using Pearson correlation test. The correlation coefficient $\mathrm{r}$ is shown when $p<0.05$. The analyses included the control group which showed the full FMA score $(F M A=100)$.

CP: cerebral peduncle; ALIC: anterior limb of the internal capsule; PLIC: posterior limb of the internal capsule; RIC: retrolenticular part of the internal capsule; CR: corona radiata; SLF: superior longitudinal fasciculus; SFOF: superior fronto-occipital fasciculus (could be a part of the anterior internal capsule); L: left; R: right. Sagittal stratum (includes the inferior longitudinal fasciculus and inferior fronto-occipital fasciculus).

\section{Figures}




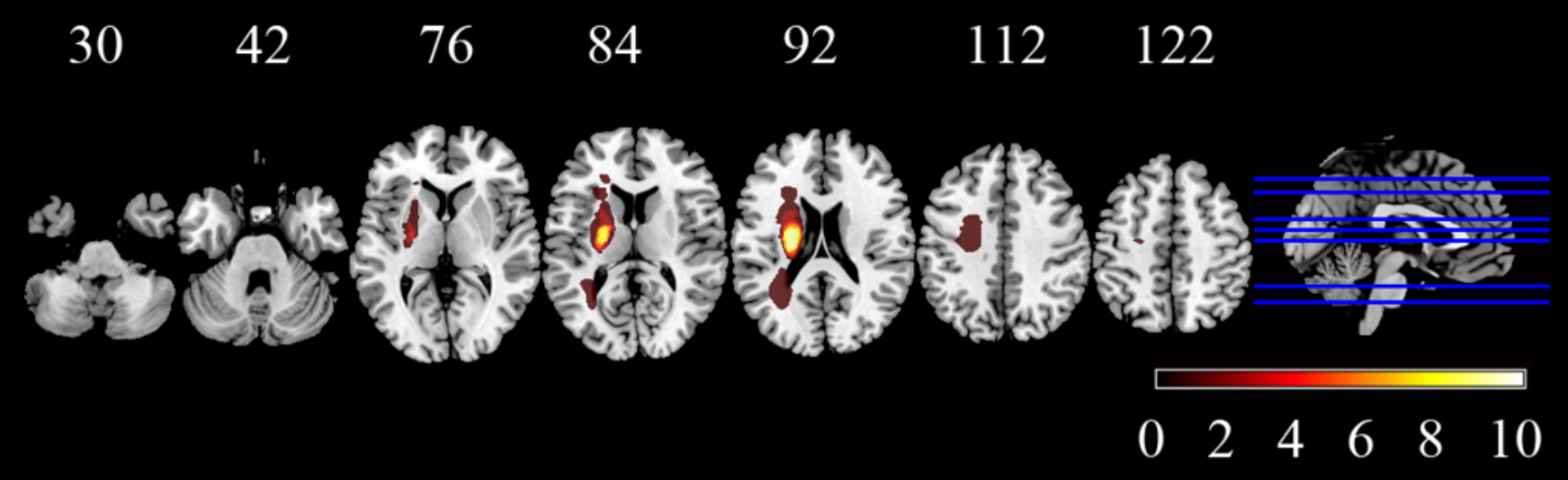

Figure 1

Lesion incidence map of patients with stroke. Stroke lesions were projected to the left hemisphere for each patient and overlaid onto a T1 template in MNI standard space. The color bar indicates the number of patients with stroke lesions in the corresponding voxel. The numbers above the brain images are $Z$ values marking the MNI coordinates of the transverse sections.

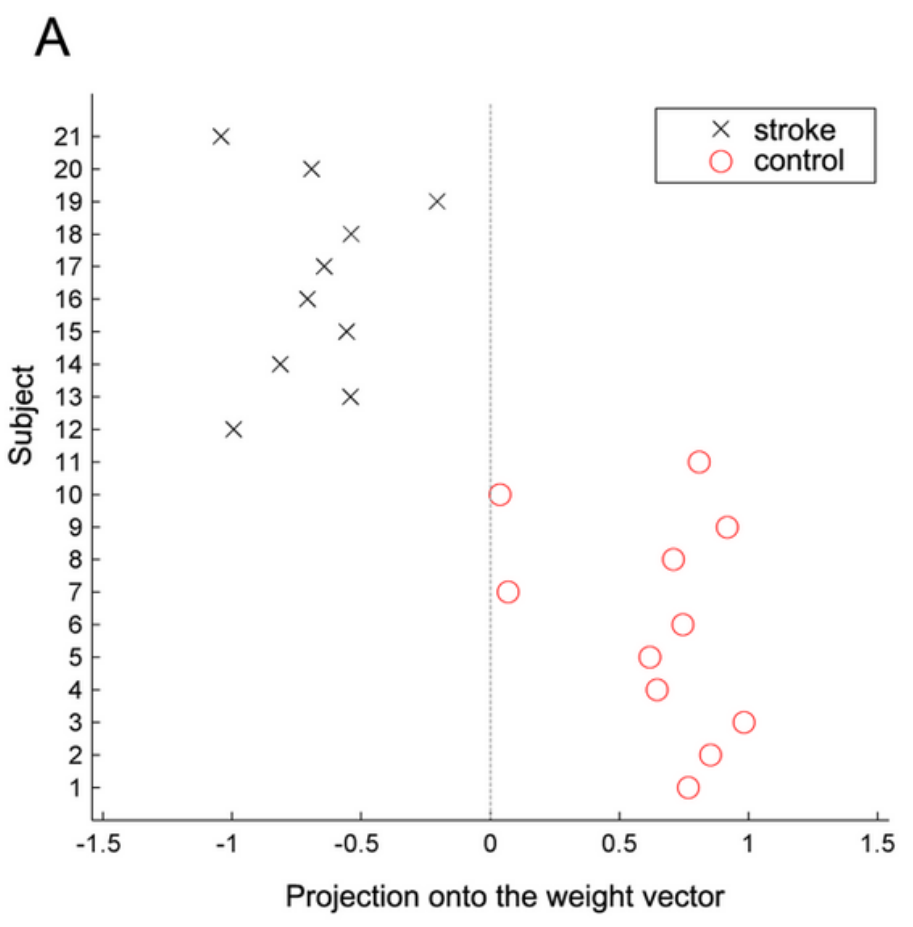

B Receiver Onerator Curve/Area Under Curve=1.00

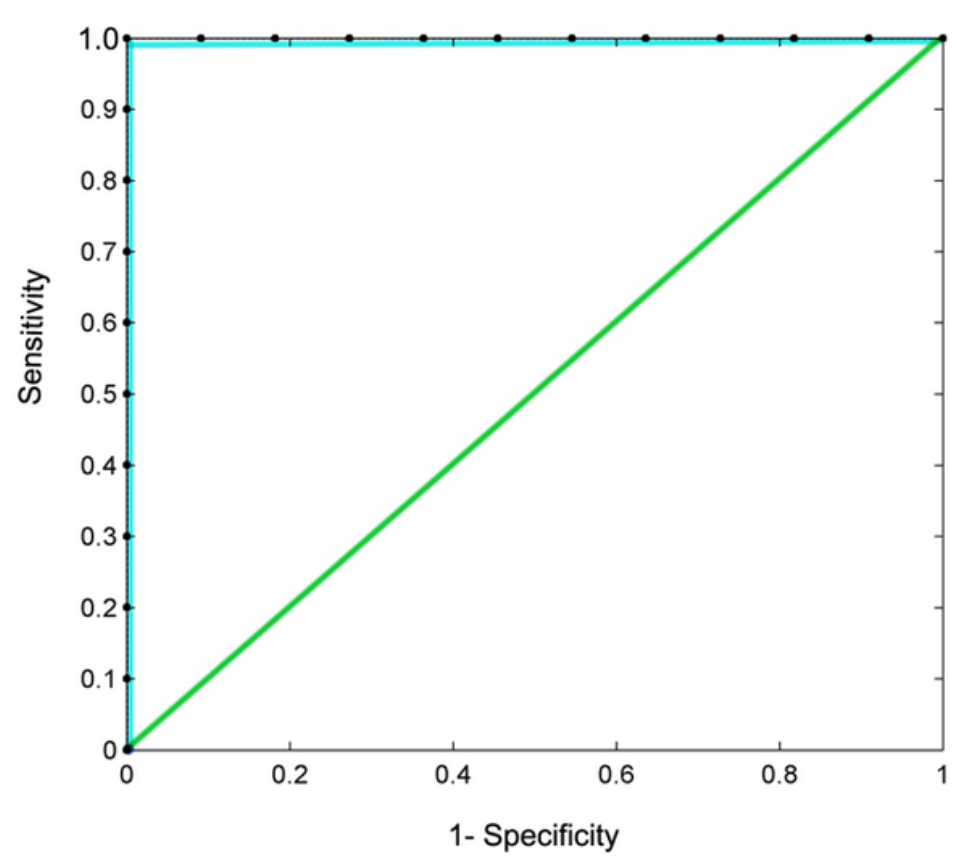

\section{Figure 2}

The results of MVPA classification. The classification plot (A) and receiver operating characteristic (ROC) curve (B) for the comparison between stroke patients and controls using FA maps derived from DTI data, 
which yielded an accuracy of 100\% (100\% sensitivity, 100\% specificity), with statistical significance at P < 0.005 .

\section{Wights map}
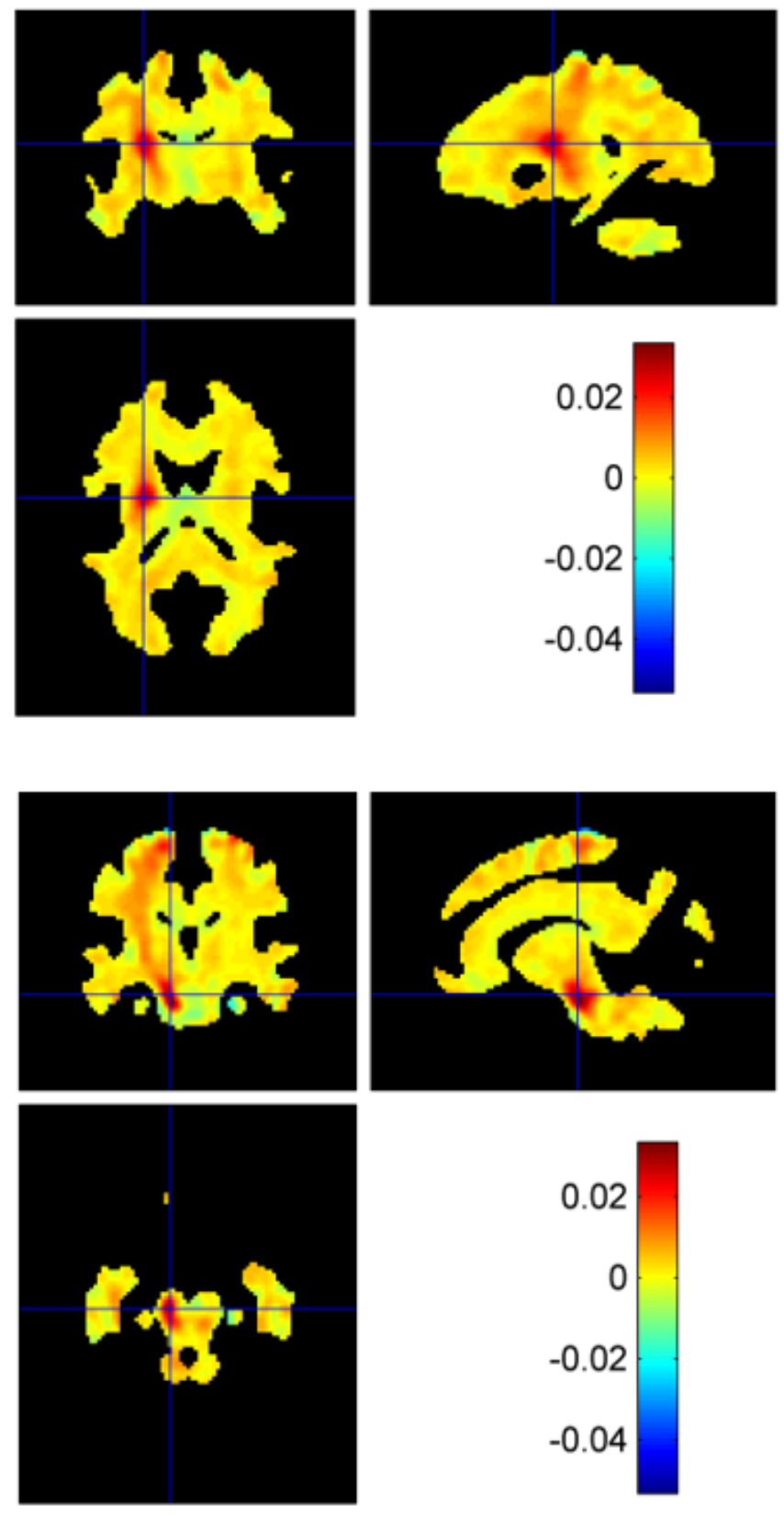

\section{Anatomical image}
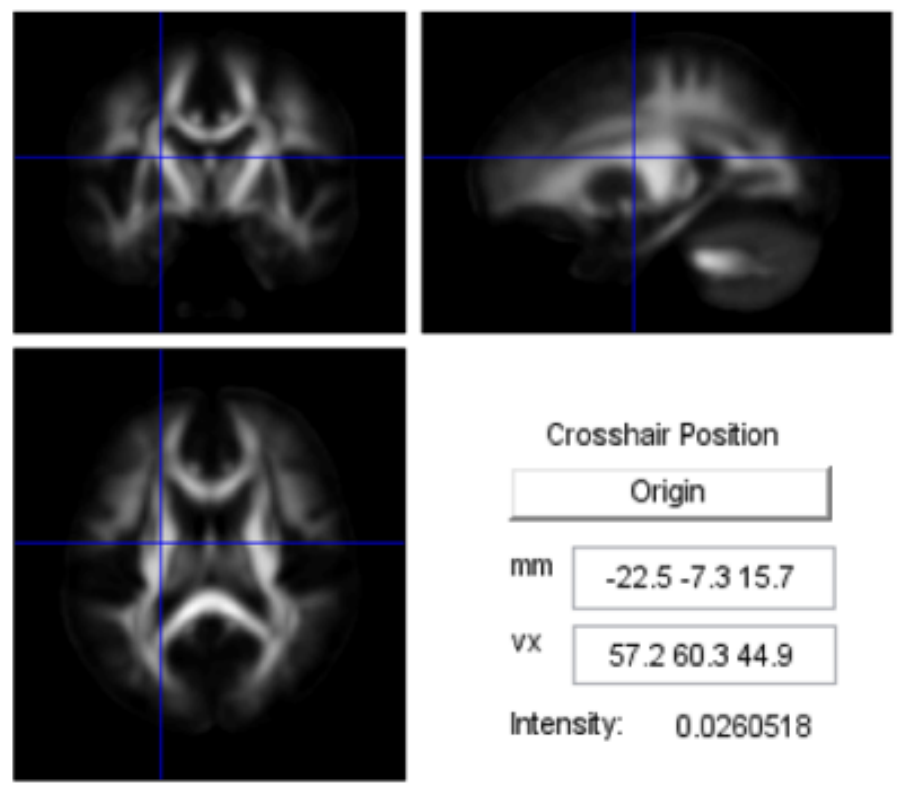

Crosshair Position

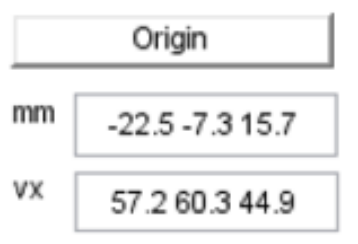

Intensity: $\quad 0.0260518$
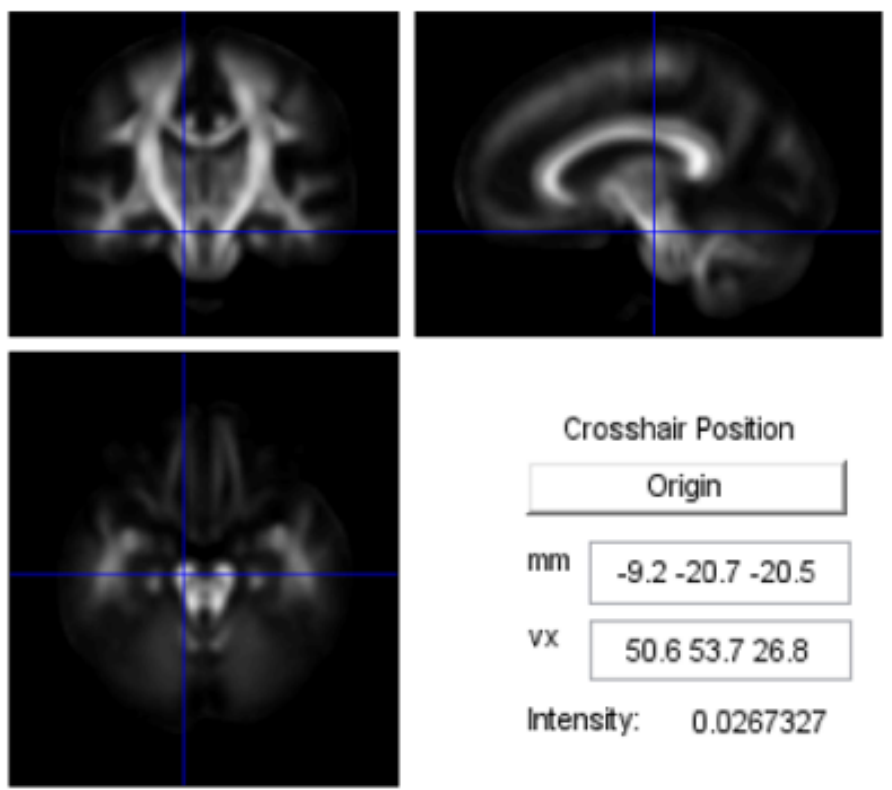

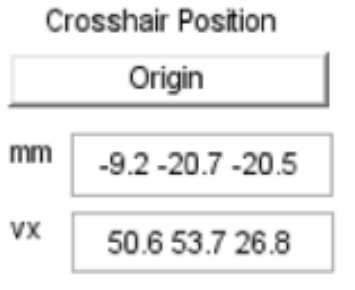

Intensity: $\quad 0.0267327$

\section{Figure 3}

Whole-brain voxel weight map. It shows the white matter regions contributing to discrimination between groups based on FA values. The color bar indicates the weight vector value of the voxel, which is also indicated in the intensity field of the Anatomical image (white fiber atlas 'JHU-ICBM-FA-2 mm') panel. 
A
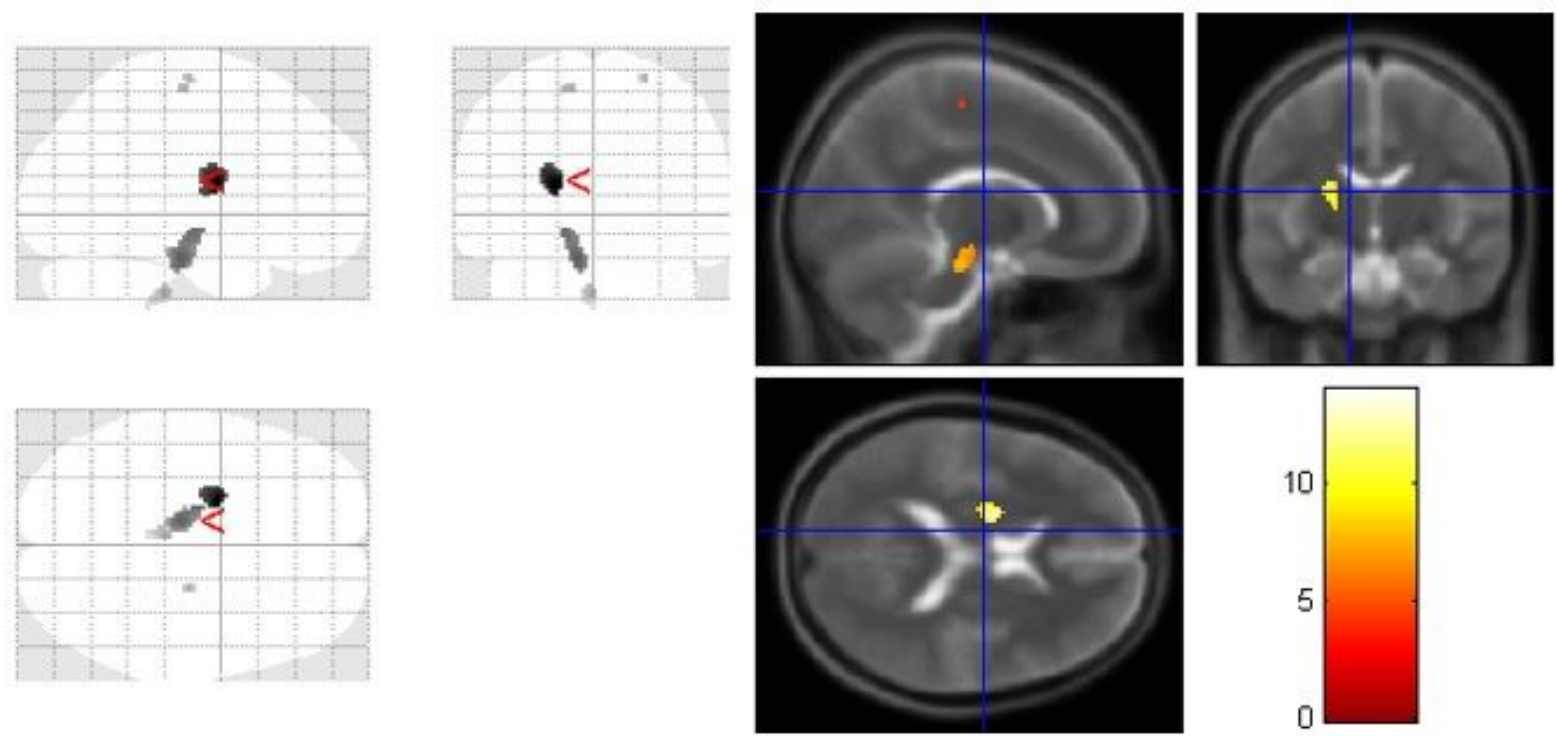

B

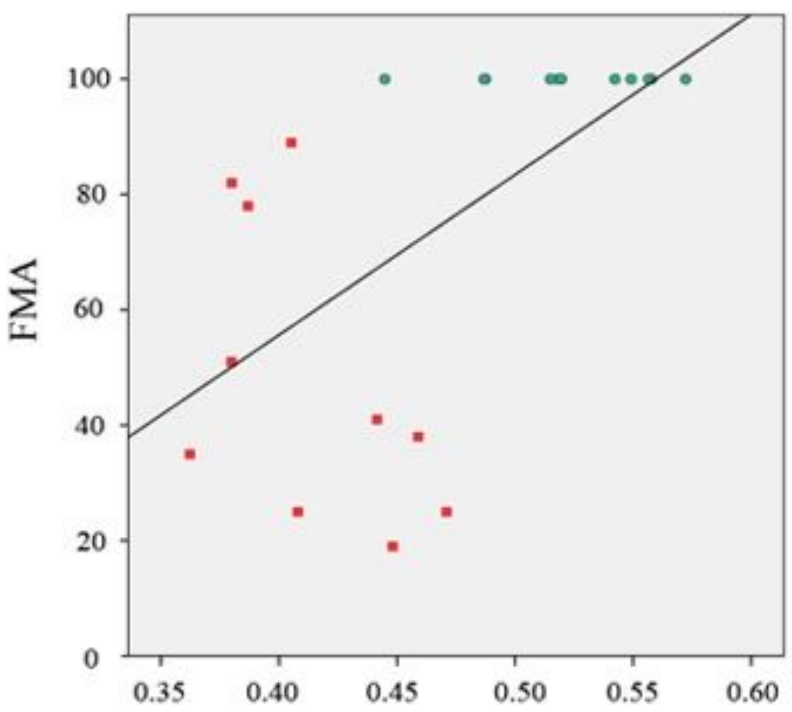

FA of CST_L

C

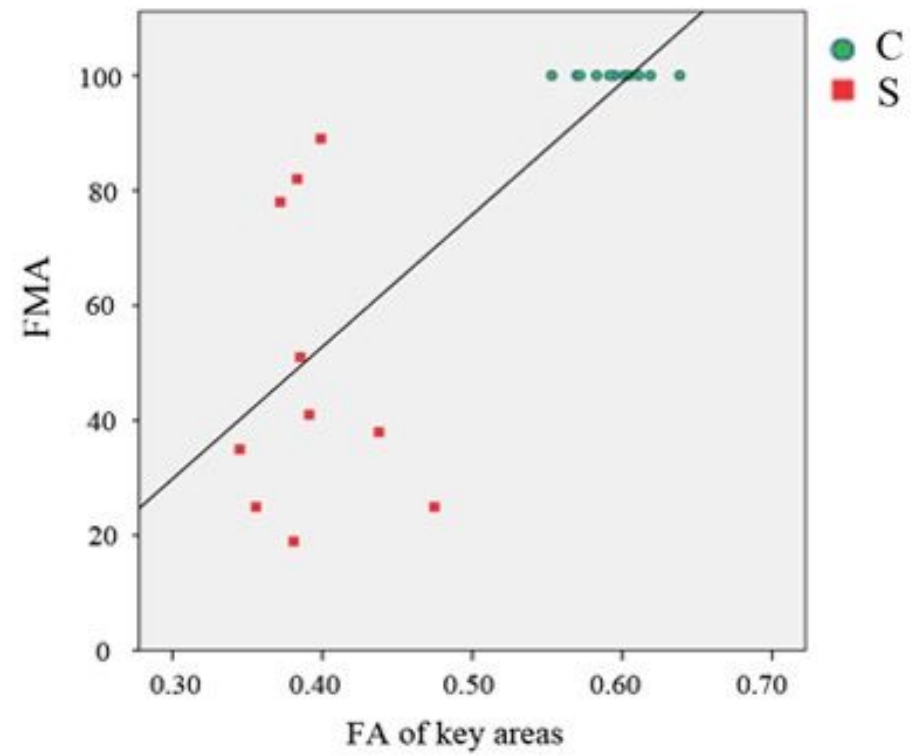

Figure 4

(A) The brain areas showing decreased FA in the patients compared to the controls, including the left brainstem, left basal ganglion and a fraction of voxels in the bilateral frontal lobe. These regions were identified by setting the threshold to $\geq 30 \%$ of the maximum weight vector scores on the basis of the whole-brain voxel weight map. The color bar indicates the T value in two-sample t tests. (B and C) The FA values of the ipsilesional CST and the key areas both showed positive correlations with FMA $(r=0.588, p$ $\leq 0.005 ; r=0.784, p<0.001)$. C: control group; $S$ : stroke group. 

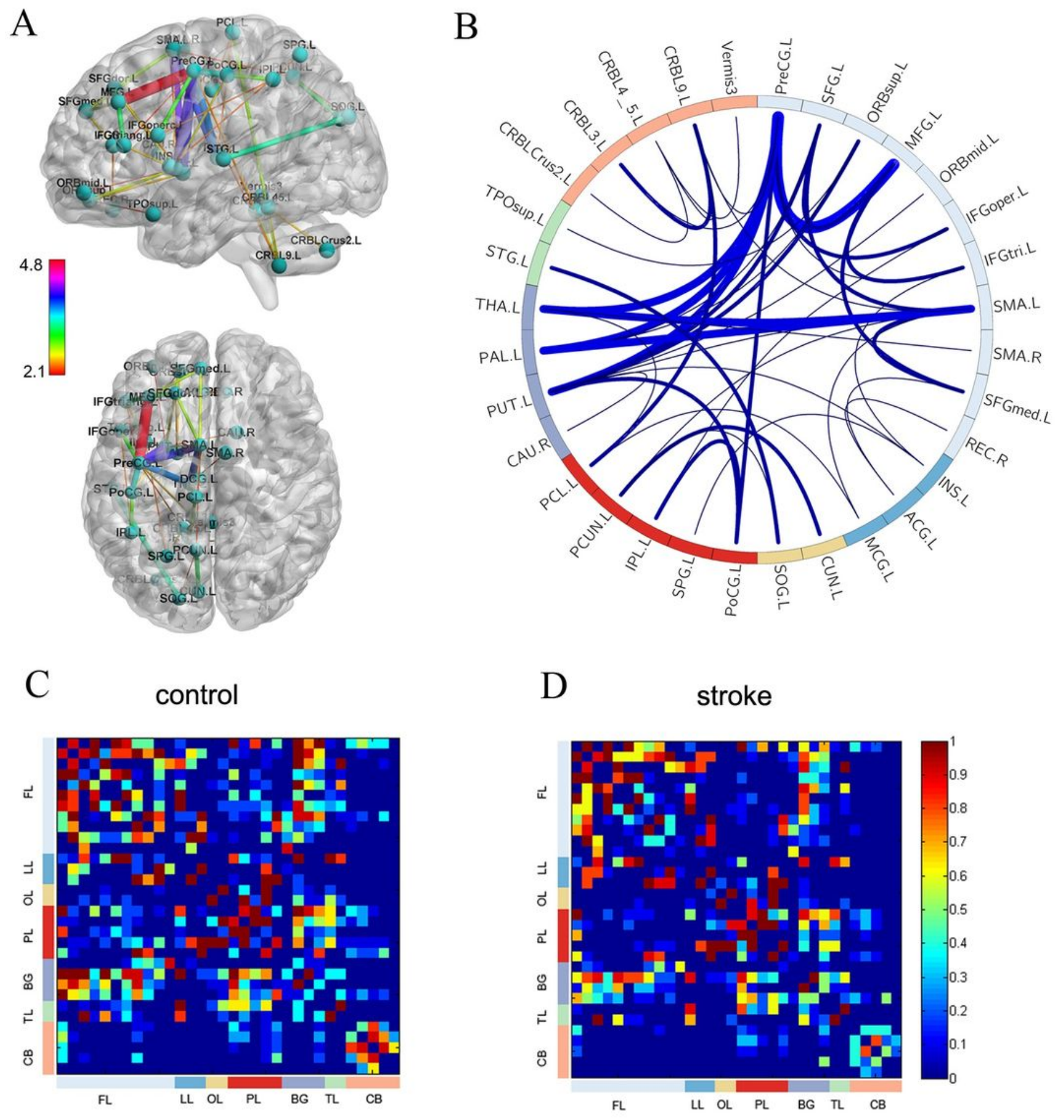

\section{Figure 5}

The subnetwork identified by the network-based statistics (NBS) analysis. (A and B) The subnetwork demonstrated reduced connectivity in stroke patients in comparison with controls. The connections in the subnetwork shown in a brain model (A) and a circle (B). The color bar represents the t value derived from two-sample t-test for each connection and the thickness of the edges represents how significantly the two groups are different (thickness of lines in $B$ : $t<2.5$, thin; $2.5 \leq t<3.5$, moderate; $t \geq 3.5$, thick). (C and $D$ ) The group averaged FA-weighted structural connectivity network for the control and stroke groups. The 
color bar indicates the connection probability in the groups. The abbreviations in B are illustrated in the Supporting Information. FL, frontal lobe; LL, limbic lobe; OL, occipital lobe; PL, parietal lobe; BG, basal ganglia; TL, temporal lobe; $\mathrm{CB}$, cerebellum.

\section{Supplementary Files}

This is a list of supplementary files associated with this preprint. Click to download.

- SupportingInformationFINALCLEANCOPY.docx 